\title{
Neutron-Antineutron Transition Search at HFIR Reactor
}

\author{
Yuri A. Kamyshkov \\ Department of Physics, University of Tennessee, Knoxville, TN 37996 \\ and Physics Division, Oak Ridge National Laboratory, Oak Ridge, TN $37891^{1}$
}

\begin{abstract}
A new experiment to search for neutron-antineutron transitions was recently proposed for High Flux Isotope Reactor (HFIR) at Oak Ridge National Laboratory (ORNL). In this paper the physics motivation of a new search, the scheme and the discovery potential of the proposed HFIR-based experiment are discussed.
\end{abstract}

\section{Physics Motivation}

Baryon asymmetry of the universe [1] and the ideas of unification of particle and forces $[2,3]$ are the two global concepts which motivated the experimental searches [4] of baryon instability for more than two decades. Neutronantineutron transitions, first considered in [5-7] within the context of these concepts and violating the baryon number by two units $(\Delta B=2)$, may be a phenomenon preferred by nature, which is alternative or complementary to the proton decay $(\Delta B=1)$. The most recent reviews of theoretical and experimental situation related to baryon instability search can be found in [8]. The experimental status of proton decay search was also discussed by T. Haines [9] at this conference.

Possible nonconservation of the baryon number is closely related to the nonconservation of lepton number. Thus, for example, in the proton decay, the conservation of angular momentum (spin of proton) requires that at least one lepton should be present in the final state. This creates in general two possibilities corresponding to $\Delta B=\Delta L$ and $\Delta B=-\Delta L$; the first one conserving $(B-L)$ and the second one violating $(B-L)$ by two units. In neutronantineutron transition, since leptons are not involved, the $(B-L)$ is violated by two units.

1) managed by Lockheed Martin Energy Research Corp. for the U.S. Department of Energy under contract number DE-AC05-960R22464.

The submitted manuscript has been authored by a contractor of the U.S. Government under contract No. DE-AC05-96OR22464. Accordingly, the U.S. 


\section{DISCLAIMER}

This report was prepared as an account of work sponsored by an agency of the United States Government. Neither the United States Government nor any agency thereof, nor any of their employees, make any warranty, express or implied, or assumes any legal liability or responsibility for the accuracy, completeness, or usefulness of any information, apparatus, product, or process disclosed, or represents that its use would not infringe privately owned rights. Reference herein to any specific commercial product, process, or service by trade name, trademark, manufacturer, or otherwise does not necessarily constitute or imply its endorsement, recommendation, or favoring by the United States Government or any agency thereof. The views and opinions of authors expressed herein do not necessarily state or reflect those of the United States Government or any agency thereof. 


\section{DISCLAMERR}

Portions of this document may be illegible in electronic image produets. Images are produced from the best available original document. 
The original $S U(5)$ unification model [3], where $(B-L)$ was conserved, favored the proton decay mode $p \rightarrow e^{+}+\pi^{0}$ with predicted lifetime $\tau / \mathrm{B}<$ $4 \cdot 10^{31}$ years. This model has been ruled out by the experiments where $\tau / B$ was measured to be $>10^{33}$ years $[8,9]$. This situation raises a question whether the $(B-L)$ in general is conserved and motivates the experimental searches for $(B-L)$ nonconserving processes. There are also several other reasons to believe that $(B-L)$ might be not conserved in nature.

It was shown [10] that in baryogenesis the nonperturbative Standard Model effects at electroweak energy scale erase any baryon excess generated at the early moments of the universe $(T \gg 1 \mathrm{TeV})$ through $(B-L)$ conserving processes. At the same time, generating baryon excess through electroweak effects alone does not seem to be adequate to account for the observed baryon asymmetry and the dark matter $[11,12]$. Thus, a component with $\Delta(B-L) \neq$ 0 might be required to explain the baryogenesis [13].

Standard Model weak interactions are not left-right symmetric like all other interactions: electromagnetic, strong, and gravitational. It is natural to think that the restoration of left-right symmetry should take place before the ultimate unification of all fundamental interaction can occur. In left-right symmetric unification models $[2,14]$ the left-right symmetry is broken at the intermediate energy scale simultaneously with $(B-L)$ violation $[15,7]$. In such models the transitions $\mathrm{n} \rightarrow \overline{\mathrm{n}}$, as well as other $(B-L)$ nonconserving processes, might exist with the rates attainable by the modern experiments [16].

The smallness of neutrino masses in theory is explained by see-saw mechanism (see discussion in [13] ) which implies the existence of heavy right-handed Majorana masses. Majorana neutrinos would violate both $L$ and $(B-L)$ by two units. Atmospheric neutrino data [17] and LSND results [18], both calling for rather large $\Delta m^{2}$ values in neutrino oscillation scenario, would require the Majorana masses and the $(B-L)$ nonconservation energy scale to be in an intermediate energy range.

Observation of "atmospheric neutrino anomaly" [17] allows an interpretation [19] of measured excess of $e^{ \pm}$in the detectors as an observation of a proton decay into the mode $p \rightarrow e^{+}+\nu+\nu .(B-L)$ is not conserved in such a decay. The "observed" rate of events corresponds to the proton lifetime of $\tau / \mathrm{B} \approx 4 \cdot 10^{31}$ years and indicates the energy scale of $\sim 10^{5} \mathrm{GeV}$ from where this process is originating.

An interesting possibility which might lead to the alternative mechanism of baryon (and $B-L$ ) number violating processes has been recently discussed by V. Kuzmin [20]. He assumed that interactions of quarks inside the baryons consisting of quarks from the different families (bus for example) might be mediated by the family-colored triplet scalar field coupled to the right components of the quarks. For neutral baryons such scalar field interaction will result in baryon-antibaryon (bus $\rightarrow \bar{b} \bar{u} \bar{s}$ ) oscillations with the characteristic time $\sim 10^{-12} \mathrm{~s}$. Such transitions can be searched at B-factories. The $\mathrm{n} \rightarrow \overline{\mathrm{n}}$ transitions in this model would arise as radiative corrections to this 
new interaction with additional suppression by $\sim 20$ orders of magnitude in probability. This will result in $\mathrm{n} \rightarrow \overline{\mathbf{n}}$ characteristic transition time of $\tau_{\bar{n} \overline{\mathbf{n}}} \sim 10^{8}$ s, i.e, close to the existing experimental limits. As mentioned in [20], the 4jet events, observed by ALEPH collaboration at LEP-II, which are peaked at $\sim 105 \mathrm{GeV}$, produced with rather high cross section, and have no signature of b-quark jets in the final state, can be explained by this model as a 3-rd component family-colored scalar-antiscalar production.

The arguments, presented above, although allowing the alternative interpretations, let us think that the $(B-L)$ might not be conserved and the energy scale corresponding to $(B-L)$ nonconservation can be as low as $\sim 10^{5}-10^{6}$ $\mathrm{GeV}$. Possible phenomena related to $\Delta(B-L) \neq 0$ would include: proton decay into modes $N \rightarrow l+$ mesons and $N \rightarrow l l \bar{l}+$ (mesons); Majorana masses for the neutrinos; neutrinoless double beta decay; transitions bus $\rightarrow \bar{b} \bar{u} \bar{s}$; intranuclear transitions of two nucleons into pions; and $n \rightarrow \bar{n}$ transitions. The question of whether such physics exists can be answered only experimentally. If it does exist, the experimental observation of $n \rightarrow \bar{n}$ transitions with free neutrons in a new reactor experiment will be its most clear and spectacular manifestation since (a) the detection signal for $\mathrm{n} \rightarrow \overline{\mathrm{n}}$ transition is clean and unambiguous and (b) the discovery potential for $n \rightarrow \overline{\mathbf{n}}$ search can be experimentally advanced by a factor of $\sim 1,000$ relative to the present experimental limits [21].

\section{Neutron-Antineutron Transition Search}

The $\mathrm{n} \rightarrow \overline{\mathrm{n}}$ transitions can be searched (a) by utilizing free neutrons from reactors or neutron spallation sources and (b) with neutrons bound inside the nuclei.

The yield of antineutrons $N_{\bar{n}}$ in the beam of free neutrons in vacuum (in the absence of external fields) due to $\mathbf{n} \rightarrow \overline{\mathbf{n}}$ transitions depends on the observation time $t$ as [7] $N_{\bar{n}} \propto N_{n} \cdot\left(t / \tau_{\mathrm{n} \bar{n}}\right)^{2}$, where $N_{n}$ is the number of neutrons used in an experiment and $\tau_{\bar{n} \bar{n}}$ is the characteristic $n \rightarrow \bar{n}$ transition time. It is assumed in this expression that neutrons and antineutrons have equal masses (as required by CPT theorem) and that the gravitational interaction with earth is the same for neutrons and antineutrons. In this way the discovery potential of an $\mathrm{n} \rightarrow \overline{\mathrm{n}}$ search experiment is proportional to the neutron flux and to the square of the neutron time-of-flight. High steady-flux reactors together with cold neutron moderators, which slow down the velocities of neutrons, would be, therefore, most appropriate for an $\mathrm{n} \rightarrow \overline{\mathrm{n}}$ search.

The general scheme of such an experiment is the following: neutrons emitted from the cold moderator are propagating in the vacuum volume (shielded against earth's magnetic field down to the level of a few $n T$ ) where the $\mathrm{n} \rightarrow \overline{\mathrm{n}}$ transition occurs. Produced antineutrons propagating along the initial neutron path would be detected as a few-meson star with total energy release 
of $\sim 1.8 \mathrm{GeV}$ resulting from the antineutron annihilation with $\mathrm{a}$ thin target film.

The recent most advanced experimental search for $\mathbf{n} \rightarrow \overline{\mathbf{n}}$ with free neutrons was performed [22] at the $58 \mathrm{MW}$ research reactor at the Institute LaueLangevin (ILL) in Grenoble. The experiment had a discovery potential of $N_{n} t^{2} \sim 1.5 \cdot 10^{9}$ seconds and for one year of operation set a limit of $\tau_{\mathrm{nn}} \geq$ $8.6 \cdot 10^{7} \mathrm{~s}$.

Intranuclear transition time $\tau_{A}$ is related to free neutron transition time $\tau_{\mathrm{n} \overline{\mathrm{n}}}$ as $\tau_{A}=T_{R} \cdot\left(\tau_{\mathrm{nn}}\right)^{2}$, where $T_{R}$ is the nuclear suppression factor. This factor has been evaluated by several authors during the last two decades. Most recent discussions and new reevaluations, as well as references to the previous works, can be found in [8]. According to [23], for oxygen, argon, and iron the suppression factor has a value $T_{R} \sim 2 \cdot 10^{23} \mathrm{~s}^{-1}$.

Experimentally, the $\mathbf{n} \rightarrow \overline{\mathbf{n}}$ transitions have been searched in the nucleon stability experiments IMB, Kamiokande, and Fréjus [21]. For example, the limit for the intranuclear $\mathbf{n} \rightarrow \overline{\mathbf{n}}$ transition lifetime for iron nuclei set by Fréjus experiment is $\tau_{\text {Iron }} \geq 6.5 \cdot 10^{31}$ years which, according to the suppression factor from [23], corresponds to $\tau_{\mathrm{nn}} \geq(0.8-1.0) \cdot 10^{8} \mathrm{~s}$.

During the next decade, the large new detectors SuperKamiokande and Icarus might improve the intranuclear $\mathbf{n} \rightarrow \overline{\mathbf{n}}$ transition limit. Thus, after a few years of operation the SuperKamiokande detector commissioned in April 1996 will be able to set an $\mathrm{n} \rightarrow \overline{\mathrm{n}}$ transition limit of $\tau_{\text {Oxygen }} \geq 10^{33}$ years [24].

The relative potentials of different methods of $n \rightarrow \bar{n}$ search were discussed in [25]. A new approach to intranuclear baryon instability search via the detection of traces of long-lived technetium isotopes in the deep-mined ores was presented by Yu. Efremenko at this conference [26].

The scheme and the discovery potential for a new proposed experiment with free neutrons at the HFIR reactor at ORNL are discussed below.

\section{A New HFIR-Based Experiment}

A new experiment for $\mathrm{n} \rightarrow \overline{\mathrm{n}}$ search at 100 MW HFIR reactor was proposed by a UT-ORNL group [27]. Proposed improvement in the discovery potential [28] is based on the properties of neutrons to be focused by means of reflection from the surfaces of certain materials. In this new approach an elliptical shape reflector intercepts the neutrons emitted from the cold source within a large solid angle and focuses them on the annihilation target situated at the distance 200-250 $\mathrm{m}$ from the source.

Schematic layout of the proposed HFIR-based experiment is illustrated in Figure 1. The gain in discovery potential (relative to the ILL-based experiment [22]) will result from the following factors: higher reactor power, larger area of the cold neutron emitting source, larger area of the annihilation detector, but, most essentially, from the use of a large-acceptance elliptical focusing 


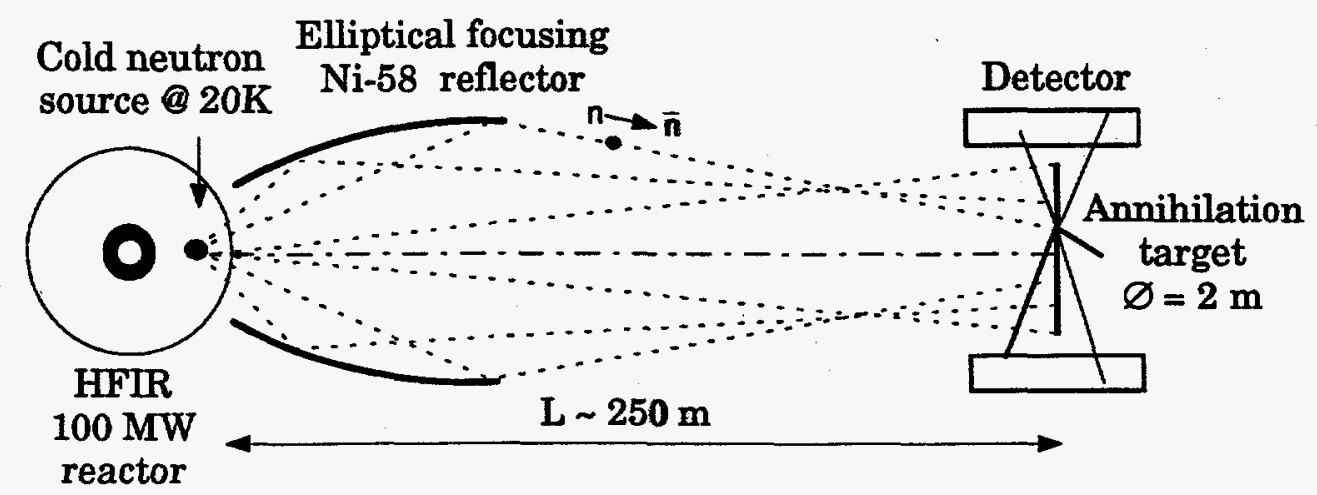

FIGURE 1. Conceptual layout of the experiment with a large elliptical focusing reflector for an $\mathbf{n} \rightarrow \overline{\mathbf{n}}$ transition search at the HFIR reactor (not to scale).

reflector. For three years of operation at HFIR the discovery potential can be increased by a factor of $\sim 1,000$ relative to the present experimental level.

\section{Conclusions}

The prospects of an $\mathbf{n} \rightarrow \overline{\mathbf{n}}$ transition search with free neutrons in reactor experiments and in intranuclear nucleon stability search experiments are compared in Figure 2. The HFIR reactor-based experiment should allow for three years of operation to increase the discovery potential of $n \rightarrow \bar{n}$ transition search by a factor of $\sim 1,000$ or to set the limit for free $n \rightarrow \bar{n}$ transition time $\geq 3 \cdot 10^{9} \mathrm{~s}$. This will explore the stability of nuclear matter, although only in one particular mode of $\mathrm{n} \rightarrow \overline{\mathrm{n}}$ transitions, to the intranuclear transition lifetime limit of $\sim 10^{35}$ years which is not attainable by other baryon instability search experiments.

If $\mathbf{n} \rightarrow \overline{\mathbf{n}}$ transition would be found, it will establish a new phenomenon leading to a new physics at the energy scale of $\sim 10^{5} \mathrm{GeV}$, i.e., beyond the range of colliders. The new symmetry principles determining the history of the universe during the first moments of creation might be revealed; the left-right symmetry, broken in the Standard Model, may be restored. The discovery of $\mathrm{n} \rightarrow \overline{\mathrm{n}}$ transition might provide a major steering impact to the unification models and contribute to the understanding of baryon asymmetry in the universe. If and when such phenomenon is established, subsequent experiments with $\mathrm{n} \rightarrow \overline{\mathrm{n}}$ transition should allow, according to [29], to perform a most precision test of CPT invariance and the test of gravitational equivalence of baryonic matter and antimatter.

I would like to thank W. M. Bugg, Yu. V. Efremenko, V. A. Kuzmin, R. N. Mohapatra, and C. D. West for useful and stimulating discussions. 


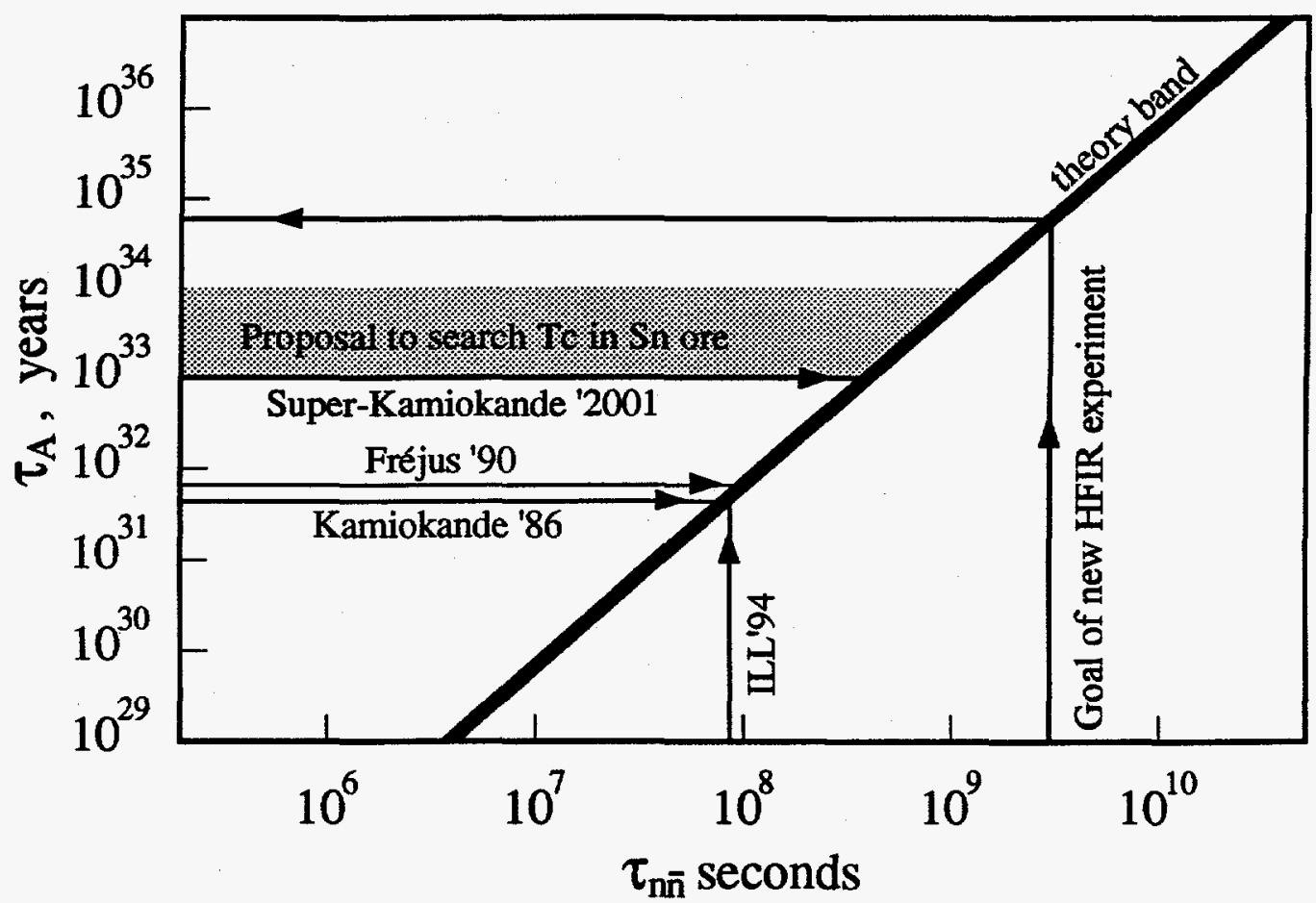

FIGURE 2. Comparison of $\mathbf{n} \rightarrow \overline{\mathbf{n}}$ search in intranuclear transitions $\left(\tau_{A}\right)$ to those in free neutron experiments $\left(\tau_{n \bar{n}}\right)$. The slope and the width of the nuclear model band connecting these two processes corresponds to $\tau_{A}=T_{R} \cdot\left(\tau_{\mathrm{n} \tilde{n}}\right)^{2}$, where $T_{R}$ is the nuclear suppression factor taken from [23].

\section{REFERENCES}

1. Sakharov A., JETP Lett. 5 (1967) 24.

2. Pati J., and Salam A., Phys. Rev. D10, 275 (1973).

3. Georgi H., and Glashow S., Phys. Rev. Lett. 32, 438 (1974).

4. Goldhaber M., "Search for Nucleon Instability (Origin and History)", in Proceedings of International Workshop on Future Prospects of Baryon Instability Search in $p$-Decay and $n \rightarrow \bar{n}$ Oscillation Experiments, Oak Ridge, 1 (1996).

5. Kuzmin V., JETP Lett. 12, 228 (1970).

6. Glashow S., preprint HUTP-79/A059.

7. Mohapatra R., and Marshak R., Phys. Lett. 91B, 222 (1980); Phys. Rev. Lett. 44, 1316 (1980); Phys. Lett. 94B, 183 (1980);

8. Proceedings of International Workshop on Future Prospects of Baryon Instability Search in p-Decay and $n \rightarrow \bar{n}$ Oscillation Experiments, Oak Ridge (1996).

9. Haines T., Overview of Proton Decay, these proceedings.

10. Kuzmin V., Rubakov V., and Shaposhnikov M., Phys. Lett. 155B, 36 (1985).

11. Rubakov V., and Shaposhnikov M., Phys. Usp. 39, 461 (1996). 
12. Kuzmin V., preprint hep-ph/9701269 (1996).

13. Pati J., in Proceedings of International Workshop on Future Prospects of Baryon Instability Search in p-Decay and $n \rightarrow \bar{n}$ Oscillation Experiments, Oak Ridge, 7 (1996); preprint hep-ph/9611371 (1996).

14. Mohapatra R., and Pati J., Phys. Rev D11, 2558 (1975).

15. Davidson A., Phys. Rev. D20, 776 (1979).

16. Mohapatra R., in Proceedings of International Workshop on Future Prospects of Baryon Instability Search in p-Decay and $n \rightarrow \bar{n}$ Oscillation Experiments, Oak Ridge, 73 (1996); preprint hep-ph/9604414 (1996).

17. Haines T., SuperKamiokande Results, these proceedings; Clark R., The Atmospheric Muon Neutrino Fraction Above $1 \mathrm{GeV}$, these proceedings; M. Goodman New Soudan $\nu_{\mu} / \nu_{e}$ Results, these proceedings.

18. Mills G., Results from the LSND Decay in Flight Oscillation Search, these proceedings.

19. Mann W.A., in Proceedings of International Workshop on Future Prospects of Baryon Instability Search in p-Decay and $n \rightarrow \bar{n}$ Oscillation Experiments, Oak Ridge, 175 (1996).

20. Kuzmin V., in Proceedings of International Workshop on Future Prospects of Baryon Instability Search in p-Decay and $n \rightarrow \bar{n}$ Oscillation Experiments, Oak Ridge, 89 (1996); preprint hep-ph/9609253 (1996).

21. Particle Data Group, "Review of Particle Physics", Phys. Rev D54, 1 (1996).

22. Baldo-Ceolin M., et al., Z. Phys C63, 409 (1994).

23. Alberico W., in Proceedings of International Workshop on Future Prospects of Baryon Instability Search in $p$-Decay and $n \rightarrow \bar{n}$ Oscillation Experiments, Oak Ridge, 221 (1996).

24. Stone J., in Proceedings of International Workshop on Future Prospects of Baryon Instability Search in p-Decay and $n \rightarrow \bar{n}$ Oscillation Experiments, Oak Ridge, 357 (1996).

25. Kamyshkov Yu., Nuclear Physics B (Proc. Suppl.) 52A, 263 (1997).;

Kamyshkov Yu., in Proceedings of International Workshop on Future Prospects of Baryon Instability Search in p-Decay and $n \rightarrow \bar{n}$ Oscillation Experiments, Oak Ridge, 281 (1996).

26. Efremenko Yu., Baryon Instability Search with Long-Lived Isotopes, these proceedings;

also Efremenko Yu., et al., in Proceedings of International Workshop on Future Prospects of Baryon Instability Search in p-Decay and $n \rightarrow \bar{n}$ Oscillation Experiments, Oak Ridge, 307 (1996).

27. Bugg W., et al., Letter of Intent to the Oak Ridge National Laboratory to Search for the $n \rightarrow \bar{n}$ Transition Using a Detector to be Built at ORNL's High Flux Isotope Reactor, UTK-PHYS-96-L1 (1996).

28. Kamyshkov Yu., in the Proceedings of the ICANS-XIII meeting, PSI, Villigen, 843 (1995).

29. Okun L., "Test of CPT", preprint ITEP-TH-55/96; hep-ph/9612247 (1996). 